\title{
Correlation for Growth, Quality, Yield and Yield Components in Yardlong Bean (Vigna unguiculata (L.) walp. ssp. Sesquipedalis verdc.)
}

\author{
P. Pratyusha Bhagavati*, T.S.K.K. Kiran Patro, N. Vara Prasad, \\ M. Lakshmi Narayana Reddy, N. Emmanuel and D.R. Salomi Suneetha
}

College of Horticulture, Dr. YSR Horticultural University, Venkataramannagudem-534101, West Godavari, Andhra Pradesh, India

*Corresponding author

\begin{tabular}{|c|c|}
\hline & A B S T R A C T \\
\hline Keywords & \multirow{4}{*}{$\begin{array}{l}\text { The present investigation was conducted on yardlong bean to identify the characters which } \\
\text { mainly contribute to the pod yield. Twenty four genotypes of yardlong bean were } \\
\text { evaluated during kharif, } 2017 \text { and observations recorded on growth, pod yield and quality } \\
\text { parameters. Correlation studies revealed that pod yield per plant }(\mathrm{g}) \text { had significant and } \\
\text { positive association with traits like vine length }(0.424 \mathrm{P}, 0.521 \mathrm{G}) \text {, number of primary } \\
\text { branches per plant }(0.862 \mathrm{P}, 0.977 \mathrm{G}) \text {, number of nodes per plant }(0.390 \mathrm{P}, 0.442 \mathrm{G}) \text {, days } \\
\text { to first flowering }(0.182 \mathrm{P}, 0.236 \mathrm{G}) \text {, length of harvesting period }(0.584 \mathrm{P}, 0.915 \mathrm{G}) \text {, pod } \\
\text { length }(0.743 \mathrm{P}, 0.801 \mathrm{G}) \text {, pod girth }(0.887 \mathrm{P}, 1.013 \mathrm{G}) \text {, number of clusters per plant } \\
(0.902 \mathrm{P}, 1.033 \mathrm{G}) \text {, length of cluster stalk }(0.722 \mathrm{P}, 1.059 \mathrm{G}) \text {, number of pods per cluster } \\
(0.843 \mathrm{P}, 1.026 \mathrm{G}) \text {, number of pods per plant }(0.840 \mathrm{P}, 1.094 \mathrm{G}) \text {, seed number per pod } \\
(0.153 \mathrm{P}, 0.250 \mathrm{G}) \text { and } 100 \text { seed weight }(0.583 \mathrm{P}, 0.658 \mathrm{G}) \text { at phenotypic and genotypic } \\
\text { levels respectively. }\end{array}$} \\
\hline $\begin{array}{l}\text { Yardlong bean, } \\
\text { Growth, Quality, } \\
\text { Yield and } \\
\text { Correlation } \\
\text { coeffiecient }\end{array}$ & \\
\hline Article Info & \\
\hline $\begin{array}{l}\text { Accepted: } \\
\text { 04 December } 2018 \\
\text { Available Online: } \\
\text { 10 January } 2019\end{array}$ & \\
\hline
\end{tabular}

\section{Introduction}

Yardlong bean (Vigna unguiculata (L.) walp. ssp. sesquipedalis verdc. $2 \mathrm{n}=22$ ) belonging to family leguminaceae is cultivated mainly for its crisp and tender green pods which are consumed both fresh as well as in cooked form.

It is also called as asparagus bean, Chinese long bean, pea bean, string bean, snake bean, snake pea, snap pea, bodi and borboti. Yardlong beans, as the name suggests, differ from cowpea in their very slender long green beans, which have a beautiful delicate flavour. This legume is also known as poor man's meat as it is a rich and inexpensive source of vegetable protein along with vitamin $\mathrm{A}$, thiamin, riboflavin, calcium, phosphorus, sodium, potassium, magnesium, vitamin $\mathrm{C}$ and micronutrients like iron, zinc, manganese and cobalt (Ano and Ubochi, 2008).

Yield is a complex character and is function of components of large number of contributing characters and their interactions. 
A study of correlation between different quantitative characters provides an idea of association and it could be effectively exploited to formulate selection strategies for improving growth, yield and quality.

\section{Materials and Methods}

The present investigation entitled "Studies on genetic variability in yardlong bean (Vigna unguiculata (L.) walp. ssp. sesquipedalis verdc.) was conducted during kharif, 2017-18 at College of Horticulture, Venkataramannagudem. Twenty four genotypes of yardlong bean collected from various places were sown in Randomized Block Design with 3 replications. Each genotype of a replication consists of eight plants, sown in two rows with a spacing of $2 \mathrm{~m}$ between the rows and $1 \mathrm{~m}$ between the plants.

All the package of practices was followed as per the recommendation.

Data pertaining to the characters such as vine length $(\mathrm{cm})$, number of primary branches per plant, number of nodes per plant, terminal leaf breadth $(\mathrm{cm})$, terminal leaf length $(\mathrm{cm})$, days to first flowering, days to $50 \%$ flowering, days to first harvest, length of harvesting period, pod length $(\mathrm{cm})$, pod girth $(\mathrm{mm})$, number of clusters per plant, length of cluster stalk, number of pods per cluster, number of pods per plant, seed number per pod, ascorbic acid content (mg/100g), TSS ( ${ }^{\circ}$ Brix), protein content $(\mathrm{mg} / 100 \mathrm{~g})$, titrable acidity $(\%), 100$ seed weight, pod yield per plant $(\mathrm{kg})$, pod yield per plot $(\mathrm{kg})$ and pod yield per hectare (tonnes) were collected from 5 randomly selected plants from each plot in each replication and subjected to statistical analysis.

Phenotypic and genotypic correlation coefficients between different variables were calculated by using covariance technique (AlJibouri et al., 1958).

\section{Results and Discussion}

The association of pod yield with other characters was estimated by genotypic and phenotypic correlation coefficient (Table 1).

Pod yield per plant (g) showed positive and significant correlation with traits like vine length $(0.424 \mathrm{P}, 0.521 \mathrm{G})$, number of primary branches per plant $(0.862 \mathrm{P}, 0.977 \mathrm{G})$, number of nodes per plant $(0.390 \mathrm{P}, 0.442 \mathrm{G})$, days to first flowering $(0.182 \mathrm{P}, 0.236 \mathrm{G})$, length of harvesting period $(0.584 \mathrm{P}, 0.915 \mathrm{G})$, pod length $(0.743 \mathrm{P}, 0.801 \mathrm{G})$, pod girth $(0.887 \mathrm{P}$, $1.013 \mathrm{G})$, number of clusters per plant $(0.902$ $\mathrm{P}, 1.033 \mathrm{G})$, length of cluster stalk $(0.722 \mathrm{P}$, $1.059 \mathrm{G})$, number of pods per cluster $(0.843 \mathrm{P}$, $1.026 \mathrm{G})$, number of pods per plant $(0.840 \mathrm{P}$, $1.094 \mathrm{G})$, seed number per pod $(0.153 \mathrm{P}$, $0.250 \mathrm{G})$ and 100 seed weight $(0.583 \mathrm{P}, 0.658$ $\mathrm{G})$ at phenotypic and genotypic levels respectively.

This trait had negative significant correlation with days to first harvest $(-0.106 \mathrm{P},-0.292 \mathrm{G})$ at phenotypic and genotypic levels. These results are in consonance with the findings of Venkatesan et al., (2003) in cowpea, Chauhan et al., (2007) in Urd bean, Mishra et al., (2008) in French bean, Katiyar and Dixit (2009) in field pea, Rai et al., (2009) in Indian bean and Devendra and Nandan (2010) in dolichos bean.

In conclusion, the relationship between yield and yield contributing characters in different genotypes of yardlong bean through phenotypic and genotypic correlations suggests that number of pods per plant and cluster stalk length $(\mathrm{cm})$ were the most important characters as they exhibited high correlation coefficient on pod yield per plant (g).

Therefore, selection for these characters would give better response. 
Table.1 Phenotypic (P) and genotypic (G) correlation coefficients among yield and yield attributes in twenty four genotypes of yardlong bean

\begin{tabular}{|c|c|c|c|c|c|c|c|c|c|c|c|c|c|c|c|c|c|c|c|c|c|c|c|}
\hline $\begin{array}{l}\text { chara } \\
\text { cters }\end{array}$ & & VL & PB & TLB & TLL & $\mathbf{N N}$ & DFF & D50 & DFH & LHP & PL & PG & NCP & CSL & NPC & NPP & NSP & AAC & TSS & PC & TA & SW & PYP \\
\hline \multirow[b]{2}{*}{ VL } & $\mathrm{P}$ & 1.000 & $0.333 * *$ & $0.345^{* *}$ & $0.443 * *$ & $0.719 * *$ & 0.133 & 0.065 & -0.150 & -0.074 & $0.416^{* *}$ & 0.450 ** & $0.449 * *$ & $0.246^{*}$ & $0.472 * *$ & $0.409 * *$ & 0.162 & 0.177 & -0.097 & 0.089 & -0.114 & $0.492 * *$ & $0.424 * *$ \\
\hline & G & 1.000 & $0.364 * *$ & $0.444^{* *}$ & $0.631^{* *}$ & $0.944^{* *}$ & 0.200 & 0.122 & $-0.233^{*}$ & -0.141 & $0.469 * *$ & $0.518^{* *}$ & $0.490 * *$ & $0.489 * *$ & $0.521^{* *}$ & $0.508^{* * *}$ & 0.156 & 0.194 & -0.093 & 0.103 & -0.176 & $0.551 * *$ & 0.521 ** \\
\hline \multirow[b]{2}{*}{ PB } & $\mathrm{P}$ & & 1.000 & -0.039 & 0.079 & $0.232^{*}$ & 0.088 & 0.023 & -0.208 & $0.634 * *$ & 0.620 ** & $0.837^{* *}$ & $0.887 * *$ & $0.701 * *$ & $0.782^{* *}$ & $0.808 * *$ & 0.219 & 0.019 & 0.148 & -0.024 & 0.091 & $0.488^{* * *}$ & $0.862^{* *}$ \\
\hline & G & & 1.000 & -0.021 & 0.209 & $0.309 * *$ & 0.188 & 0.079 & $-0.251^{*}$ & $0.973 * *$ & $0.667 * *$ & $0.938^{* *}$ & $0.983^{* *}$ & $1.021 * *$ & 0.920 ** & $1.044 * *$ & $0.434 * *$ & 0.016 & 0.172 & -0.039 & 0.132 & $0.514 * *$ & $0.977^{* * *}$ \\
\hline \multirow[b]{2}{*}{ TLB } & $\mathrm{P}$ & & & 1.000 & $0.806^{* *}$ & $0.441^{* *}$ & 0.214 & $0.249 *$ & 0.112 & -0.216 & 0.106 & -0.019 & -0.019 & -0.016 & -0.043 & 0.029 & -0.005 & 0.178 & -0.035 & 0.037 & 0.052 & 0.041 & -0.027 \\
\hline & G & & & 1.000 & $0.896^{* *}$ & $0.596^{* *}$ & $0.318 * *$ & $0.384 * *$ & 0.158 & $-0.374 * *$ & 0.116 & -0.074 & -0.006 & 0.084 & -0.043 & -0.074 & 0.148 & 0.212 & 0.054 & 0.035 & 0.006 & 0.025 & -0.020 \\
\hline \multirow[b]{2}{*}{ TLL } & $P$ & & & & 1.000 & $0.417^{* *}$ & 0.215 & 0.201 & 0.084 & -0.112 & 0.097 & 0.175 & 0.134 & 0.033 & 0.135 & 0.156 & $0.254^{*}$ & 0.112 & 0.050 & 0.073 & 0.020 & 0.086 & 0.090 \\
\hline & G & & & & 1.000 & $0.774 * *$ & $0.269^{*}$ & $0.281^{*}$ & 0.163 & $-0.277^{*}$ & 0.163 & 0.181 & 0.190 & 0.292* & 0.198 & 0.155 & $0.517^{* *}$ & 0.179 & 0.077 & 0.101 & -0.082 & 0.134 & 0.176 \\
\hline \multirow[b]{2}{*}{$\mathbf{N N}$} & $P$ & & & & & 1.000 & 0.107 & 0.048 & -0.162 & -0.136 & $0.438^{* *}$ & $0.385^{* *}$ & $0.354 * *$ & $0.282^{*}$ & $0.363^{* *}$ & $0.330^{* * *}$ & 0.061 & $0.393 * *$ & -0.085 & 0.121 & -0.062 & $0.461 * *$ & 0.390 ** \\
\hline & G & & & & & 1.000 & 0.121 & 0.023 & $-0.379 * *$ & -0.141 & $0.520 * *$ & $0.462 * *$ & 0.432 ** & $0.383^{* * *}$ & $0.485^{* *}$ & $0.448 * *$ & 0.209 & $0.448 * *$ & -0.020 & 0.139 & -0.120 & $0.588^{* * *}$ & $0.442 * *$ \\
\hline \multirow[b]{2}{*}{ DFF } & $\mathrm{P}$ & & & & & & 1.000 & 0.945 & $0.744 * *$ & -0.049 & 0.155 & 0.177 & 0.179 & 0.093 & 0.123 & 0.181 & -0.080 & 0.214 & -0.084 & $-0.278^{*}$ & 0.066 & 0.155 & 0.182 \\
\hline & G & & & & & & 1.000 & 0.964 & $0.768^{* * *}$ & 0.037 & 0.202 & $0.266^{*}$ & $0.233 *$ & 0.343 & $0.295^{*}$ & 0.221 & $-0.456^{* *}$ & $0.299^{*}$ & -0.221 & -0.447 ** & -0.003 & 0.208 & $0.236^{*}$ \\
\hline \multirow[b]{2}{*}{ D50 } & $\mathrm{P}$ & & & & & & & 1.000 & $0.776^{* *}$ & -0.081 & 0.076 & 0.076 & 0.085 & 0.056 & -0.005 & 0.102 & -0.089 & 0.197 & -0.086 & $-0.296^{*}$ & 0.116 & 0.098 & 0.078 \\
\hline & G & & & & & & & 1.000 & $0.827 * *$ & -0.060 & 0.083 & 0.095 & 0.118 & $0.262^{*}$ & 0.126 & 0.068 & $-0.425 * *$ & $0.287^{*}$ & $-0.248^{*}$ & $-0.492 * *$ & 0.057 & 0.140 & 0.081 \\
\hline \multirow[b]{2}{*}{ DFH } & P & & & & & & & & 1.000 & -0.114 & -0.066 & -0.124 & -0.146 & -0.156 & -0.216 & -0.095 & -0.029 & 0.098 & -0.040 & $-0.313 * *$ & -0.049 & -0.116 & -0.106 \\
\hline & G & & & & & & & & 1.000 & -0.145 & -0.157 & -0.212 & -0.228 & -0.126 & -0.216 & -0.303 ** & $-0.411 * *$ & 0.104 & -0.167 & $-0.535 * *$ & -0.120 & -0.163 & -0.291 \\
\hline \multirow[b]{2}{*}{ LHP } & $\mathrm{P}$ & & & & & & & & & 1.000 & $0.432 * *$ & $0.576^{* *}$ & $0.604 * *$ & $0.451 * *$ & $0.658^{* *}$ & $0.607 * *$ & -0.036 & -0.042 & 0.108 & -0.004 & 0.151 & 0.198 & $0.584 * *$ \\
\hline & G & & & & & & & & & 1.000 & $0.599 * *$ & $0.872^{* *}$ & $0.912 * *$ & $1.062 * *$ & $0.752^{* *}$ & $0.936 * *$ & $0.331 * *$ & -0.062 & $0.365^{* *}$ & 0.003 & 0.045 & $0.316 * *$ & $0.915^{* * *}$ \\
\hline \multirow[b]{2}{*}{ PL } & $\mathrm{P}$ & & & & & & & & & & 1.000 & $0.682 * *$ & $0.727 * *$ & 0.543 ** & $0.662^{* *}$ & $0.679 * *$ & -0.098 & $0.389 * *$ & -0.002 & 0.072 & -0.006 & 0.716 ** & $0.743^{* *}$ \\
\hline & G & & & & & & & & & & 1.000 & $0.770^{* *}$ & $0.795^{* *}$ & $0.859^{* *}$ & $0.737^{* *}$ & $0.816^{* * *}$ & -0.084 & $0.402 * *$ & -0.003 & 0.082 & -0.032 & $0.751^{* * *}$ & 0.801 ** \\
\hline \multirow[b]{2}{*}{ PG } & $\mathrm{P}$ & & & & & & & & & & & 1.000 & $0.901 * *$ & $0.633^{* *}$ & $0.868^{* *}$ & $0.816^{* *}$ & 0.157 & 0.221 & 0.098 & 0.059 & -0.061 & $0.539 * *$ & $0.887 * *$ \\
\hline & G & & & & & & & & & & & 1.000 & $1.000^{* *}$ & $1.099 * *$ & $1.018^{* *}$ & $1.080^{* *}$ & 0.297* & 0.215 & 0.170 & 0.054 & -0.018 & $0.621 * *$ & $1.013^{* * *}$ \\
\hline \multirow[b]{2}{*}{ NCP } & $\mathrm{P}$ & & & & & & & & & & & & 1.000 & $0.722^{* *}$ & $0.885^{* *}$ & $0.796 * *$ & 0.182 & 0.176 & 0.095 & 0.023 & 0.025 & $0.597 * *$ & 0.902 *** \\
\hline & G & & & & & & & & & & & & 1.000 & $1.073^{* *}$ & $0.972 * *$ & $1.129 * *$ & $0.308^{* *}$ & 0.190 & 0.115 & 0.037 & 0.067 & $0.678^{* *}$ & $1.033^{* * *}$ \\
\hline \multirow[b]{2}{*}{ CSL } & $\mathrm{P}$ & & & & & & & & & & & & & 1.000 & $0.606^{* *}$ & $0.598^{* *}$ & 0.070 & 0.185 & 0.119 & 0.061 & -0.015 & $0.414 * *$ & $0.722 * *$ \\
\hline & G & & & & & & & & & & & & & 1.000 & $1.091^{* *}$ & $1.199 * *$ & $0.478^{* *}$ & $0.259^{*}$ & 0.093 & 0.121 & 0.007 & $0.694 * *$ & $1.059^{* *}$ \\
\hline
\end{tabular}




\begin{tabular}{|c|c|c|c|c|c|c|c|c|c|c|c|c|c|c|c|c|c|c|c|c|c|c|c|}
\hline $\begin{array}{c}\text { charact } \\
\text { ers }\end{array}$ & & $V L$ & PB & TLB & TLL & $\mathrm{NN}$ & DFF & D50 & DFH & LHP & $\mathrm{PL}$ & PG & NCP & CSL & NPC & NPP & NSP & AAC & TSS & PC & TA & SW & PYP \\
\hline \multirow[b]{2}{*}{ NPC } & $\mathrm{P}$ & & & & & & & & & & & & & & 1.000 & $0.788^{* *}$ & 0.105 & 0.110 & 0.081 & 0.101 & 0.002 & $0.554^{\star *}$ & $0.843^{*}$ \\
\hline & G & & & & & & & & & & & & & & 1.000 & $1.083^{* *}$ & $0.305^{\star *}$ & 0.125 & 0.205 & 0.127 & -0.072 & $0.634^{* *}$ & $1.026^{*}$ \\
\hline \multirow[b]{2}{*}{ NPP } & $P$ & & & & & & & & & & & & & & & 1.000 & 0.118 & 0.150 & 0.040 & 0.033 & 0.020 & $0.564^{* *}$ & $0.840^{* *}$ \\
\hline & G & & & & & & & & & & & & & & & 1.000 & $0.382^{* *}$ & 0.155 & 0.197 & 0.060 & 0.035 & $0.678^{* *}$ & $1.094^{*}$ \\
\hline \multirow[b]{2}{*}{ NSP } & $\mathrm{P}$ & & & & & & & & & & & & & & & & 1.000 & -0.063 & $0.268^{*}$ & 0.206 & -0.086 & 0.071 & 0.153 \\
\hline & G & & & & & & & & & & & & & & & & 1.000 & -0.135 & $0.278^{*}$ & $0.249^{*}$ & -0.181 & 0.214 & $0.250^{*}$ \\
\hline \multirow[b]{2}{*}{ AAC } & $P$ & & & & & & & & & & & & & & & & & 1.000 & $-0.381^{\star *}$ & 0.140 & 0.135 & $0.493^{* *}$ & 0.176 \\
\hline & G & & & & & & & & & & & & & & & & & 1.000 & $-0.467^{* *}$ & 0.134 & 0.165 & $0.525^{\star \star}$ & 0.185 \\
\hline \multirow[b]{2}{*}{ TSS } & $P$ & & & & & & & & & & & & & & & & & & 1.000 & -0.041 & -0.039 & -0.173 & 0.098 \\
\hline & G & & & & & & & & & & & & & & & & & & 1.000 & -0.048 & -0.171 & -0.224 & 0.117 \\
\hline \multirow[b]{2}{*}{ PC } & $P$ & & & & & & & & & & & & & & & & & & & 1.000 & 0.064 & $0.255^{*}$ & 0.046 \\
\hline & G & & & & & & & & & & & & & & & & & & & 1.000 & 0.078 & $0.283^{*}$ & 0.040 \\
\hline \multirow[b]{2}{*}{ TA } & $\mathrm{P}$ & & & & & & & & & & & & & & & & & & & & 1.000 & 0.129 & 0.003 \\
\hline & G & & & & & & & & & & & & & & & & & & & & 1.000 & 0.156 & 0.064 \\
\hline \multirow[b]{2}{*}{$100 S$} & $\mathrm{P}$ & & & & & & & & & & & & & & & & & & & & & 1.000 & $0.583^{*}$ \\
\hline & G & & & & & & & & & & & & & & & & & & & & & 1.000 & $0.658^{*}$ \\
\hline \multirow[b]{2}{*}{ PYP } & $P$ & $0.424^{* *}$ & $0.862^{* *}$ & -0.027 & 0.090 & $0.390^{* *}$ & 0.182 & 0.078 & -0.106 & $0.584^{* *}$ & $0.743^{* *}$ & $0.887^{* *}$ & $0.902^{* *}$ & $0.722^{* *}$ & $0.843^{* *}$ & $0.840^{* *}$ & 0.153 & 0.176 & 0.098 & 0.046 & 0.003 & $0.583^{* *}$ & 1.000 \\
\hline & & $0.521^{* \star}$ & $0.977^{* *}$ & -0.020 & 0.176 & $0.442^{\star *}$ & $0.236^{*}$ & 0.081 & -0.291 & $0.915^{* *}$ & $0.801^{* *}$ & $1.013^{* *}$ & $1.033^{* *}$ & $1.059^{* \star}$ & $1.026^{* *}$ & $1.094^{* *}$ & $0.250^{*}$ & 0.185 & 0.117 & 0.040 & 0.064 & $0.658^{* *}$ & 1.000 \\
\hline
\end{tabular}

\section{*significant at $5 \%$ LOS * significant at $1 \%$ LOS}

$\mathrm{VL}=$ Vine Length $(\mathrm{cm}) ; \mathrm{NPB}=$ Number of Primary branches $; \mathrm{TLL}=$ Leaf length $(\mathrm{cm}) ; \mathrm{TLB}=$ Leaf breadth $(\mathrm{cm}) ; \mathrm{NN}=\mathrm{Number}$ of nodes per plant $; \mathrm{DF}=\mathrm{Days}$ to first flowering ; DFF = Days to 50\% flowering ; DFH=Days to first harvesting ; LHP=Length of harvesting period (days) ; PL= Pod length ; PG= Pod girth ; $\mathrm{NCP}=$ No. of clusters per plant $; \mathrm{CSL}=$ Cluster stalk length $(\mathrm{cm}) ; \mathrm{NPC}=$ No. of pods per cluster $; \mathrm{NPP}=$ Number of pods per plant $; \mathrm{SNP}=\mathrm{Seed}$ number per pod ; $\mathrm{AA}=$ Ascorbic acid content (mg/100g) ; TSS=Total soluble solids ; PC=Protein content; TA=Titrable acidity ; SW= Seed weight; PYP= Pod yield per plant 


\section{References}

Al-Jibouri, H. A., Miller, P. A and Robinson, H. F. 1958. Genotypic and environmental variation and correlation in upland cotton cross of interspecies origin. Agronomy Journal. 50: 633-37.

Ano, A. O and Ubochi, C. I. 2008. Nutrient composition of climbing and prostrate vegetable cowpea accessions. African Journal of Biotechnology. 7(20): 37953798.

Chauhan, M. P., Mishra, A. C and Singh, A. K.2007. Correlation and path analysis in urd bean. Legume Research. 30(3): 20508 .

Devendra, U and Nandan, M. 2010. Biometrical studies in dolichos bean (Dolichos lablab L.) for Chhattisgarh plains. Research Journal of Agricultural
Sciences. 1(4): 441-47.

Katiyar, P. K and Dixit, G. P. 2009. Multivariate analysis for genetic divergence in field pea (Pisum sativum) germplasm. Indian Journal of Agricultural Sciences. 79(3):181-83.

Mishra, S., Kumar, M and Sahu, G. S. 2008. Relationships among yield contributing characters in pole type French bean (Phaseolus vulgaris L.). The Orissa Journal of Horticulture. 36(2): 108-13.

Rai, N., Singh, P. K., Verma, A., Yadav, P. K and Choubey, T. 2010. Hierarchical analysis for genetic variability in pole type French bean. Indian Journal of Horticulture. 67(Special issue): 150-53.

Venkatesan, M., Prakash, M and Ganesan, J. 2003. Correlation and path analysis in cowpea [Vigna unguiculata (L.) Walp.]. Legume Research. 26(2): 105-08.

\section{How to cite this article:}

Pratyusha Bhagavati, P., T.S.K.K. Kiran Patro, N. Vara Prasad, M. Lakshmi Narayana Reddy, N. Emmanuel and Salomi Suneetha, D.R. 2019. Correlation for Growth, Quality, Yield and Yield Components in Yardlong Bean (Vigna unguiculata (L.) walp. ssp. Sesquipedalis verdc.). Int.J.Curr.Microbiol.App.Sci. 8(01): 410-414. doi: https://doi.org/10.20546/ijcmas.2019.801.042 